\title{
Numerical Simulation on the Dynamics Behavior of Non-traveling Wave Soliton to the Zhiber-Shabat Equation with Parameter Perturbation
}

\author{
Zhiqiang $\mathrm{Pu}^{1,{ }^{1}}$, Zhigang $\mathrm{Pan}^{2}$ and Xuelian Zhang ${ }^{3}$ \\ ${ }^{1}$ School of Information Engineering, Mianyang Teachers' College, Mianyang, Sichuan, 621000, PR \\ China \\ ${ }^{2}$ School of Mathematics, Southwest Jiaotong University, Chendu, Sichuan, 610000, PR China \\ ${ }^{3}$ Department of Art and Design, Sichuan Vocational College Of Art, Chendu, Sichuan, 610000, PR \\ China \\ *Corresponding author
}

Keywords: Zhiber-shabat equation, Lie symmetry group, Non-traveling wave solitons, Matlab numerical simulation.

\begin{abstract}
Study on the parameter perturbation behavior about non-traveling wave mechanics to the Zhiber-Shabat equation by Lie symmetry group precise symbolic computation and numerical simulation technology. The Lie point symmetry group and reduced of the equations are obtained. The dynamics simulation model about local excitation parameter perturbation of non-traveling wave soliton are showed by difference scheme and the Matlab numerical calculation of symmetric reduced equation with variable coefficients. The results present the scientific connotation of the new content to the equation and indicate the effectiveness of thought which combined by the symbolic computation and numerical calculation.
\end{abstract}

\section{Introduction}

Nonlinear partial differential equation is an important mathematical model for describing physical phenomena. The study of exact solutions has received extensive attention in recent decades, and many effective methods have been derived, such as backscattering transformation [1], Backlund transformation[2], Hirota bilinear[3], homogeneous balance[4], extended $\mathrm{F}$ expansion[5], Lie group[6,7] and so on.

This paper considers the following Ziber-Shabat equation[8-11]:

$$
u_{x t}+p e^{u}+q e^{-u}+r e^{-2 u}=0
$$

Where $u: R_{x} \times R_{t}^{+} \rightarrow R, p 、 q, r \in R$ and $p^{2}+q^{2}+r^{2} \neq 0$.when $q=r=0$, equation (1) is reduced to the famous Liouville equation. When $r=0$, equation (1) is reduced to the famous Sinh-Gordon equation. When $q=0$, equation (1) is reduced to the famous Dodd-Bullough-Mikhailov equation. When $p=0, q=-1, r=1$, the Tzitzeica-Dodd-Bullough equation can be obtained. The above equations play an important role in many scientific applications.

For the equations (1) [8], the hyperbolic tangent function method is used to obtain the traveling wave solutions with different physical structures[9]. The bifurcation theory and phase plane analysis method are used to study dynamic behavior of traveling wave[10].Utilization exponential function is used to obtains a new type of exact solution[11]. The F-expansion method is used to obtain the elliptic function periodic solution. Obviously, these works are the most basic work for the traveling wave solution. However, the non-traveling wave solution structure with more scientific significance and its dynamic parameter perturbation behavior have not been studied in the literature.

In this paper, the Lie group method is applied first, and the non-traveling wave of the Zhiber-Shabat equation is reduced to the variable coefficient nonlinear ordinary differential equation by symbolic calculation. Then the differential method is used to numerically calculate the 
numerical solution of the reductive system, we shall research the non- traveling wave dynamics parameter perturbation behavior of the model.

\section{Lie Point Symmetry Reduction}

Under the transformation:

$$
u(x, t)=\ln w(x, t)
$$

Then equation (1) is transformed into the following 1+1-dimensional nonlinear partial differential equation related to the function $w(x, t)$.

$$
w w_{x t}-w_{x} w_{t}+p w^{3}+q w+r=0
$$

Let $\sigma(x, t)$ is the Lie point symmetry of $w(x, t)$, according to the Lie group theory, $\sigma(x, t)$ satisfy the following equation:

$$
\sigma_{x t} w+\sigma w_{x t}-\sigma_{t} w_{x}-\sigma_{x} w_{t}+3 p \sigma w^{2}+q \sigma=0
$$

Assume $\sigma(x, t)$ has the following form:

$$
\sigma=a w_{t}+b w_{x}+c w+d
$$

Among them $a=a(x, t), b=b(x, t), c=c(x, t), d=d(x, t)$ are the pending smooth functions of the variables $x, t$, and $w(x, t)$ is the solution of the equation (3). Substituting equation (5) with equation (3) into equation (4), by linearly independent of its derivatives from $w(x, t)$, we find the solution as follows:

$$
a=-k t-v, b=k x+v, c=d=0
$$

Where $k, v \in R$, the symmetry of equation (3) is obtained as:

$$
\sigma=-(k t+v) w_{t}+(k x+v) w_{x}
$$

Solving differential equation $\sigma=0$ to obtain:

$$
w=F(\xi), \quad \xi=k x t+v(x+t)+v_{0}
$$

Where $v_{0} \in R$, putting equation (8) into equation (3), we have acquired the second-order cubic non-linear differential equation of variable coefficient:

$$
k F F^{\prime}+\left(k \xi+v^{2}\right)\left(F F^{\prime \prime}-F^{\prime 2}\right)+p F^{3}+q F+r=0
$$

The solution of this equation is an important part of exploring the non- traveling wave dynamic characteristic of Zhiber-Shabat equation (1). However, with our symbolic calculation, there are still more difficulties to overcome in order to obtain an exact solution to this equation. For this purpose, we use the difference method to find the numerical solution of equation (9) in the next paragraph, and then explore the perturbation behavior of the non-traveling wave soliton dynamic parameters of equation (1).

\section{Numerical Simulation of the Perturbation Behavior of Non- traveling Wave Soliton Dynamic Parameters for ZS Equation (1)}

\section{Finite Difference Scheme of Equation (9)}

In order to ensure the stability and convergence of the algorithm, the first-order difference operator adopts the backward difference scheme, and the second-order difference operator uses the common discrete scheme. Therefore, the backward difference scheme of equation (9) is: 


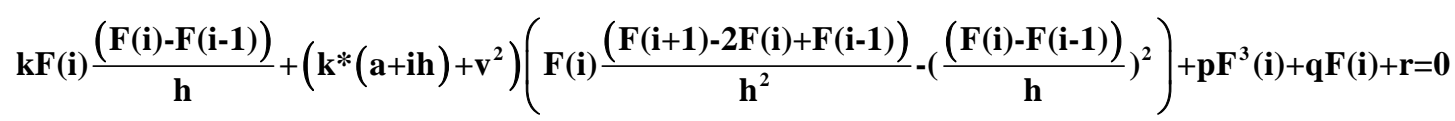

\section{The Design of Newton Iterative Solution Program for Difference Equation (10)}

Building a Difference Equation Function. The homogeneous boundary condition is taken $F(1)=0, F(N)=0$, and the interval value is $[a, b]$, where $N$ is the number of discrete points on the discrete interval, program for evaluation is designed by appling the Newton iterative algorithm based on Matlab:

function eq=myfun(F)

$\mathrm{a}=\mathrm{a} ; \mathrm{b}=\mathrm{b}$;

$\mathrm{h}=\mathrm{h}$;

$\mathrm{n}=(\mathrm{b}-\mathrm{a}) / \mathrm{h}+1 ; \quad \%$ Number of discrete points on the interval value is $[a, b]_{\%}$

$\mathrm{k}=\mathrm{k}$; \% parameter setting\%

$\mathrm{v}=\mathrm{v}$;

$\mathrm{p}=\mathrm{p}$;

$\mathrm{q}=\mathrm{q}$;

$\mathrm{r}=\mathrm{r}$;

eq(1) $=F(1)$;

for $i=2: n-1$

$\mathrm{eq}(\mathrm{i})=\mathrm{k}^{*} \mathrm{~F}(\mathrm{i}) *(\mathrm{~F}(\mathrm{i})-\mathrm{F}(\mathrm{i}-1)) / \mathrm{h}+\left(\mathrm{k}^{*}(\mathrm{a}+\mathrm{i} * \mathrm{~h})+\mathrm{v}^{\wedge} 2\right) *(\mathrm{~F}(\mathrm{i}) *(\mathrm{~F}(\mathrm{i}+1)-2 * \mathrm{~F}(\mathrm{i})+\mathrm{F}(\mathrm{i}-1)) / \mathrm{h} \wedge 2-((\mathrm{F}(\mathrm{i})-\mathrm{F}(\mathrm{i}-1)) / \mathrm{h}$

)$\left.^{\wedge} 2\right)+\mathrm{p} * \mathrm{~F}(\mathrm{i}) \wedge 2+\mathrm{q} * \mathrm{~F}(\mathrm{i})+\mathrm{r}$;

end

eq(n) $=F(n)$;

Calling the function "myfun" to solve the nonlinear difference equation by least square method(10). $\mathrm{a}=\mathrm{a} ; \mathrm{b}=\mathrm{b} ; \quad \%$ Select discrete interval $[a, b]$ for simulation

$\mathrm{h}=\mathrm{h} ; \quad \%$ Step size of interval discrete \%

$\mathrm{xi}=\mathrm{a}: \mathrm{h}: \mathrm{b}$

$\mathrm{n}=(\mathrm{b}-\mathrm{a}) / \mathrm{h}+1$;

$\mathrm{v} 0=0$;

$\mathrm{k}=\mathrm{k}$;

$\mathrm{v}=\mathrm{v}$;

$\mathrm{F} 0=$ zeros $(1, \mathrm{n})$;

$\mathrm{F}=$ fsolve('myfun',F0)

$\%$ size(F)

$\% \operatorname{plot}(\mathrm{xi}, \mathrm{F})$

$\mathrm{t}=\mathrm{a}: \mathrm{h}: \mathrm{b}$

$\mathrm{x}=\mathrm{t}$;

$[\mathrm{T}, \mathrm{X}]=$ meshgrid$(\mathrm{t}, \mathrm{x})$;

for $\mathrm{i}=1: \mathrm{n}$

$$
\begin{aligned}
& \text { for } \mathrm{j}=1: \mathrm{n} \\
& \mathrm{xxi}=\left(\mathrm{k}^{*} \mathrm{x}(\mathrm{j}) * \mathrm{t}(\mathrm{i})+\mathrm{v}^{*}(\mathrm{x}(\mathrm{i})+\mathrm{t}(\mathrm{i}))+\mathrm{v} 0\right) \text {; } \\
& \text { if }(x x i<b) \\
& \text { else } \\
& u(i, j)=0 \text {; }
\end{aligned}
$$$$
\mathrm{u}(\mathrm{i}, \mathrm{j})=\log (\operatorname{abs}(\text { interp1(xi,F,xxi,'linear'))); }
$$

end

end

$\% \operatorname{mesh}(\mathrm{T}, \mathrm{X}, \mathrm{u})$

subplot(1,2,1), plot(xi,F);subplot(1,2,2),surf(T,X,u);shading interp;view(38,81);\%axis off; 
Local Structure of Dynamics in the Solution of ZS Equation (1) and Equation (9): In order to understand the phenomena more clearly, we show three comparison figures of the equation (9) with $\alpha=1, \beta=1, p=q=r=1, a=-0.2, b=0.2, v=2, h=0.005$, (1) $k=-2$, (2) $k=0$, (3) $k=2$.

(1) $k=-2$
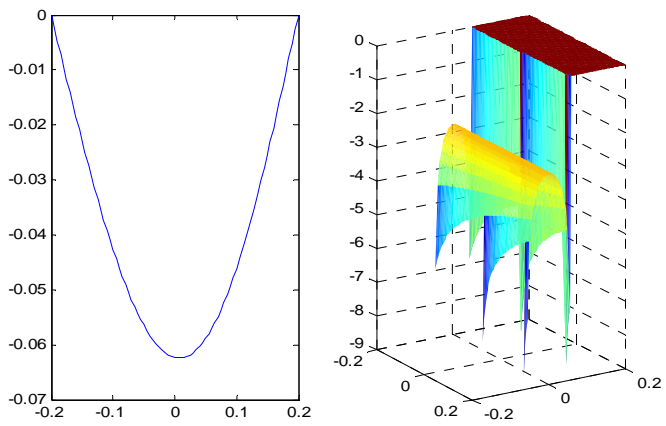

Figure 1. $k=-2$

(2) $k=0$
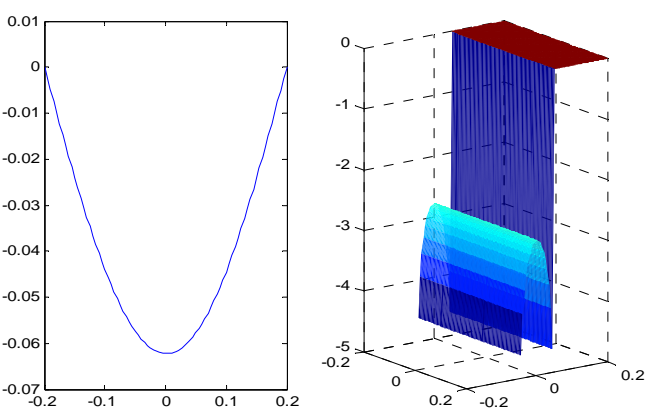

Figure 2. $k=0$

(3) $k=2$
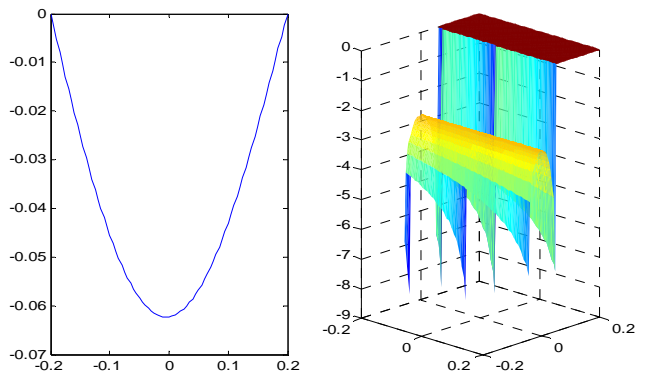

Figure 3. $k=2$

The left figure of Fig.1, Fig 2 and Fig 3 shows the nonlinear wave numerical simulation of the nonlinear ordinary differential equation (9), and the right figure of Fig.1, Fig 2 and Fig 3 shows the nonlinear wave numerical simulation of the corresponding Zhiber-Shabat equation (1). When $k=0$, it is a nonlinear traveling wave structure, as shown in figure 2 . When $k \neq 0$, it is a nonlinear non-traveling wave structure, as shown in figure 1 and 3. Form the Fig.1, Fig 2 and Fig 3, we can find that the sign of $k$ determines the local monotonicity of the evolution of solution wave. When $k>0$ the local monotonous increases(see in figure 1 ). When $k<0$ the local monotonous reduces(see in figure 3 ). 


\section{Conclusion}

In this paper, the Lie point symmetry of the Zhiber-Shabat equation is obtained by using the Lie group method, and the equation is symmetrically reduced into a nonlinear ordinary differential equation with variable coefficients. The finite difference scheme of symmetric reduction equation and the Matlab numerical solution program based on Newton iteration algorithm is given. The local geometry of the perturbation behavior of non- traveling wave soliton dynamic parameters of equation (1) is shown. The new research ideas in this paper can be used to study the perturbation mode of non- traveling wave solution dynamic behavior parameters of other mathematical physics models.

\section{Acknowledgments}

This work was supported by Chinese Natural Science Foundation Grant o.11361048, Sichuan educational Science Foundation under Grant No.12ZB069 and No.13ZB0125.

\section{References}

[1] Yomba, E, Construction of new soliton-like solutions for the $(2+1)$ dimensional Kadomtsev-Petviashvili equation. Chaos. Solitons and Fractals.21,75-79(2004)

[2] Xi-Qiang Liu, Song Jiangc, Wen-Bin Fan, Wu-Ming Liu, Soliton solutions in linear magnetic field and time-dependent laser field, Communications in Nonlinear Science and Numerical Simulation. 9,361-365 (2004)

[3] BAI Cheng-Lin, LIU Xi-Qiang and ZHAO Hong, Backlund Transformation and Multiple Soliton Solutions for (3+1)-Dimensional Potential-YTSF Equation, Commun. Theor. Phys.42,827-830(2004)

[4] Zhengde Dai, Daquan Xian, Homoclinic breather-wave solutions for Sine-Gordon equation, Communications in Nonlinear Science and Numerical Simulation. 14(8),3292-3295(2009)

[5] Mingliang Wang, Xiangzheng Li, Extended F-expansion method and periodic wave solutions for the generalized Zakharov equations. Phys.Lett.A.343,48-54(2005)

[6] YAN Zhi-Lian and LIU Xi-Qiang, Solitary Wave and Non-traveling Wave Solutions to Two Nonlinear Evolution Equations, Commun. Theor. Phys. 44,479-482(2005)

[7] Xian Da-Quan, Chen Han-Lin, Symmetry Reduced and New Exact Non-traveling Wave Solutions of Potential Kadomtsev-Petviashvili Equation with p-Power, Appl. Math. Comput. 16(1),70-79 (2009)

[8] Abdul-Majid Wazwaz, The tanh method for travelling wave solutions to the Zhiber-Shabat equation and other related equations, Communications in Nonlinear Science and Numerical Simulation, 13(3),584-592(2008)

[9] Yaning Tang, Wei Xu, Jianwei Shen, Liang Gao, Bifurcations of traveling wave solutions for Zhiber-Shabat equation, Nonlinear Analysis: Theory, Methods \& Applications, 67(2), 648-656(2007)

[10]ZHAO Yun-mei, Jl Xiao-ming, RUI W ei-guo, New exact solutions for Zhiber-Shabat equation using Exp-function method, Journal of Southwest University for Nationalities-Natural Science Edition.33(6), 1214-1219 (2007)

[11] Yunmei Zhao, Weiguo Rui,The soliton solution and the periodic wave solution of Zhiber. Shabat equation, Pure mathematics and Applied Mathematics.2(24),283-288(2008) 
[12] Yadong Shang, New exact special solutions with solitary patterns for Boussinesq-like B(m,n) equations with fully nonlinear dispersion, Applied Mathematics and Computation .173,1137-1148 (2006). 